\title{
Länger leben mit Vollkorn
}

Eine ballaststoffreiche Ernährung senkt nicht nur das Risiko für kardiovaskuläre Erkrankungen, sondern erhöht auch die Chancen auf ein langes Leben. Das ist zumindest das Ergebnis der mit 390000 Teilnehmern und einer Laufzeit von neun Jahren bisher umfassendsten prospektiven Beobachtungsstudie zu diesem Thema. In dem Fünftel der Teilnehmer mit dem höchsten Ballaststoffkonsum (Männer 29,4 $\mathrm{g} / \mathrm{d}$, Frauen $25,8 \mathrm{~g} / \mathrm{d}$ ) Lebensversicherung?

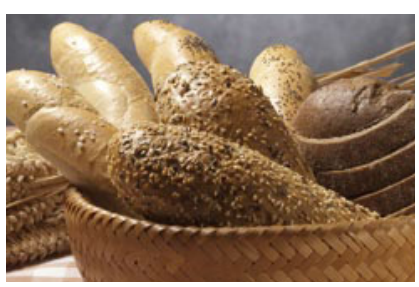

war die Sterblichkeit insgesamt um 22\% geringer. Neben einer verminderten Rate von kardiovaskulären Todesfällen gab es auch weniger Todesfälle durch respiratorische und Infektionskrankheiten. Die lebensverlängernde Wirkung war übrigens hauptsächlich auf Vollkornprodukte zurückzuführen, Obst und Gemüse trugen deutlich weniger dazu bei. Unter anderem werden antiinflammatorische Effekte als Ursache für die breite Wirkung vermutet.

Arch Intern Med, Februar 2011; doi: 10.1001/archinternmed.2011.18

\section{PROSTATAKARZINOM}

\section{Neuer Risikofaktor: Haarausfall in jungen Jahren}

Männer, die bereits im Alter von 20 Jahren zur Glatzenbildung neigen, haben ein signifikant erhöhtes Risiko, später an einem Prostatakarzinom zu erkranken. Wer früh seine Haare verliert, profitiert daher möglicherweise in besonderem Maße von der Prostatakarzinom-Früherkennung. Dies ergab eine Studie von Onkologen der Pariser Descartes Universität. In der Studie wurden

Bei Männern, deren Glatzenbildung Jahren einsetzte, fand sich kein Unterschied zur Kontrollgruppe.

Ann Onc 2011; DOI: 10.1093/annonc/mdq695

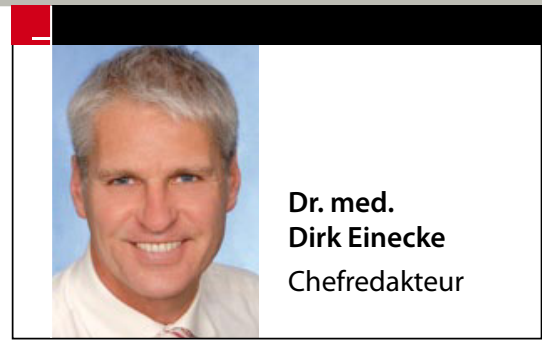

BEHANDLUNG VON SCHMERZEN

\section{Die Erwartung bestimmt die Wirkung}

Wer an die Wirksamkeit eines Schmerzmittels glaubt, spürt eine deutlich stärkere Schmerzlinderung. Das zeigt eine kleine Studie, in der Probanden einem Schmerzreiz ausgesetzt wurden und dabei per Infusion ein opioidhaltiges Schmerzmittel erhielten. Wussten die Patienten von der Behandlung, war die analgetische Wirkung stärker als bei „heimlicher" Verabreichung. Am geringsten war der Effekt, wenn die Patienten dachten, die Infusion sei bendet worden. Studienerstautorin Dr. Ulrike Bingel vom Universitätsklinikum HamburgEppendorf vermutet, dass eine negative Erwartung und die Angst vor dem Schmerz den Effekt des Opioids aufheben.

Sci Transl Med 2011, 3 (70):70ra14. DOI: 10.1126/ scitransImed.3001244

\section{SCHLAGANFALLPRÄVENTION BEI VORHOFFLIMMERN}

\section{Acetylsalicylsäure hat ausgedient}

Patienten mit Vorhofflimmern, die kein Marcumar einnehmen können oder wollen, profitieren von einem Faktor-Xa-Inhibitor mehr als von Acetylsalicylsäure. In der sog. AVERROES-Studie erhielten 5599 Patienten mit Vorhofflimmern, die nicht mit Vitamin-K-Antagonisten behandelt werden konnten, entweder Apixaban 5 mg zweimal täglich oder ASS in Dosen zwischen 81 und $344 \mathrm{mg}$ am Tag. Da sich eine signifikante Verminderung von Schlaganfällen unter dem neuen Gerinnungshemmer ergab (relative Risikominderung 55\%), wurde die Studie vorzeitig abgebrochen. Die Zahl von Blutungskomplikationen war für beide Behandlungen gleich.

Ein Vergleich zwischen Apixaban und Warfarin bei Patienten mit Vorhofflimmern steht noch aus. Die Ergebnisse der entsprechenden Studie (ARISTOTLE) werden im August erwartet.

NEJM 2011;DOI:10.1056/NEJMoa1007432
www.SpringerMedizin.de

Was Hausärzte am meisten interessiert

Diese Artikel wurden im Allgemeinmedizin-Bereich von SpringerMedizin.de in den letzten Tagen am häufigsten aufgerufen:

- 1. Missgeburt von Eminenzen? „Leitlinien wurden sicher nicht von Hausärzten erfunden!" (333220)

- 2. Dossier "Spektakuläre (Un-)Fälle (309616)

- 3. Katastrophale Essgewohnheiten: Die Deutschen "snacken" sich durch den Tag (332522)

Die Artikel finden Sie, indem Sie die ID-Nummer (in Klammern) in die Suche eingeben. 